\section{Breaking the code for AXL in metastasis}

\section{By Kai-Jye Lou, Staff Writer}

Researchers at the University of Bergen have shown that AXL receptor tyrosine kinase is a key regulator of metastasis and a strong predictor of poor overall survival in breast cancer patients. ${ }^{1}$ BerGenBio A/S has licensed the IP and is moving the work forward with target validation studies.

AXL receptor tyrosine kinase (AXL; UFO) is a member of the TAM family of receptor tyrosine kinases and has a history of associations with many aspects of tumorigenesis. Although studies have implicated AXL as a promoter of cell migration, invasiveness and disease metastasis in breast cancer, ${ }^{2-4}$ its functional link to key processes in metastasis has remained unclear.

The Bergen group now has unraveled the mechanism and shown that AXL is involved in epithelial-to-mesenchymal transitions (EMTs). Cancer cells undergoing EMT acquire improved
"AXL is not just a biomarker-our work shows that it is functionally required for the metastatic phenotype in breast cancer."

- James Lorens, University of Bergen cancer patients, and we show that in this subpopulation, the clinical outcome is much worse," said Lorens, who is the corresponding author on the paper. "AXL is not just a biomarker-our work shows that it is functionally required for the metastatic phenotype in breast cancer. There is a functional correlation between AXL and the genes associated with EMT," he said.

"AXL is an exciting therapeutic target that appears to control the progression of metastatic breast cancer by regulating tumor cell spread from the primary location as well as tumor growth in niches outside the tissue of origin," said Sacha Holland, director of biology at Rigel Pharmaceuticals Inc. "Dr. Lorens' team showed that $A X L$ expression is both essential for metastatic dissemination and a major negative determinant of survival in mouse models of metastatic breast cancer."

"The metastasis model studies in the PNAS paper were very thorough," said Jason Foulks, manager of discovery biology and head of the AXL inhibitor program at SuperGen Inc. "Previous research has not shown the association between AXL and breast cancer metastasis to this level of detail."

Foulks also noted that AXL's link to EMT is significant. "I'm intrigued by the hypothesis that AXL can be involved in a positive feedback loop with EMT. It would be important to study whether GAS6 is also required," he said.

Growth arrest-specific 6 (GAS6) is a known ligand for AXL.

Douglas Graham, an associate professor of pediatrics and immunology at the University of Colorado Denver, said the results in the migratory and survival abilities, which facilitate malignant progression and metastasis. Recent studies have implicated EMT with cancer stem cell traits and drug resistance. ${ }^{5,6}$

"We stumbled upon AXL in a screen for regulators of cell migration a few years ago, and this led us down the pathway to show that it is functionally associated with EMT," said James Lorens, vice-chairman of the Department of Biomedicine at the University of Bergen and a BerGenBio cofounder.

In mouse xenograft and cell culture studies, the Bergen group identified AXL as a key downstream effector of EMT in breast cancer cells. Small hairpin RNA-mediated knockdown of $A X L$ reduced the invasiveness of human breast cancer cells compared with that seen using control shRNA. In mouse models of human breast cancer, shRNA-mediated knockdown of $A X L$ prevented disease metastasis and increased survival.

The researchers also showed that EMT-inducing transcription factors in human breast epithelial cells led to $A X L$ expression. In a cohort of breast cancer patients, those with high $A X L$ expression had lower overall survival than patients with low $A X L$ expression $(p=0.035)$. Tissue samples taken from the patients showed that $A X L$ expression was significantly higher in metastatic lesions than in the corresponding primary tumor $(p<0.0005)$.

Results were published in the Proceedings of the National Academy of Sciences.

" $A X L$ upregulation defines a subpopulation of about $40 \%$ of breast
PNAS article make a case for evaluating the effects of therapeutics on the occurrence of metastatic lesions as a clinical trial endpoint.

Indeed, metastasis is the primary cause of breast cancer mortality in Western women. ${ }^{7}$

Rigel's R428, a small molecule inhibitor of AXL catalytic activity, is in preclinical development to treat cancer. Holland said she expects preclinical data to be published next month.

\section{An old new target}

Although $A X L$ was cloned from patients and associated with malignant transformation in 1991, 8,9 there are no disclosed AXL-specific inhibitors in the clinic.

Lorens noted that the initial lack of industry interest in AXL may be because "its main function relates to cell migration and survival, not proliferation, which has been the focus in the field. It was overlooked."

"Studies have suggested that AXL-targeting compounds as monotherapy would be more effective at circumventing resistance and preventing metastasis" but not at eliminating tumors, said SuperGen's Foulks.

SuperGen could be the first company to enter the clinic with a targeted AXL inhibitor. The biotech has small molecule AXL inhibitors in preclinical development to treat cancer and hopes to file an IND for 
its lead inhibitor in late 2010 or early 2011.

At least two tyrosine kinase inhibitors in the clinic, bosutinib and foretinib, have shown nonselective inhibition of AXL function. ${ }^{10}$ Bosutinib (SKI-606), a small molecule BCR-ABL tyrosine kinase inhibitor from Pfizer Inc.'s Wyeth unit, is in Phase III testing to treat chronic myelogenous leukemia (CML). Foretinib (GSK1363089, formerly XL880), a small molecule spectrum selective kinase inhibitor that GlaxoSmithKline plc licensed from Exelixis Inc., is in Phase II testing to treat multiple cancers.

Although SuperGen hasn't disclosed the specific indications it plans to pursue with its AXL inhibitors, Foulks thinks a good starting point could be preventing tumor metastasis or invasion in patients with high AXL expression. "The biology of AXL is still relatively new compared to the other receptor tyrosine kinases," he said. "It's still necessary to figure out how an AXL inhibitor would behave in the body."

Michael McCullar, VP of strategy and discovery operations at SuperGen, also suggested that targeting AXL could help improve the responsiveness of cancers that are resistant to both standard chemotherapy and targeted therapies.

"One key piece of information needed to advance AXL further as a therapeutic target is to develop ways to identify the subset of cancer patients that would benefit from an AXL-targeting treatment," said Graham. "It would be important to screen for patients with upregulation of $A X L$ or AXL-dependent pathways when designing clinical trials."

SuperGen's Foulks agreed that clinical trials should incorporate a method to identify patients that would be sensitive AXL inhibitors. "Targeting the right population would be very important," he said.

Indeed, the drug development pathway going forward could be challenging. Without having some method to detect and enroll at-risk patients for clinical trials, it would be difficult for researchers to demonstrate a treatment effect. Moreover, clinical trials designed to show that a compound could prevent metastasis would require large patient populations and long follow-up times. It also is usually necessary to show that a treatment has an effect on the primary tumor. ${ }^{11}$

Rigel's Holland added that "it will also be key to determine the effectiveness of AXL inhibitors in combination with the standard-ofcare therapies for target tumor types."

Lorens said his group is studying additional signaling and activation mechanisms for AXL and is developing antibodies that block its function. He told SciBX that the University of Bergen has filed for a patent covering the work and its diagnostic and therapeutic applications. Lorens said the IP is available for licensing through BerGenBio, which is evaluating the therapeutic use of AXL-targeting antibodies in cancer.

Lou, K.-J. SciBX 3(4); doi:10.1038/scibx.2010.106

Published online Jan. 28, 2010

\section{REFERENCES}

1. Gjerdrum, C. et al. Proc. Natl. Acad. Sci. USA; published online Dec. 28, 2009; doi:10.1073/pnas.0909333107

Contact: James B. Lorens, University of Bergen, Bergen, Norway e-mail: jim.lorens@biomed.uib.no

2. Holland, S.J. et al. Cancer Res. 65, 9294-9303 (2005)

3. Zhang, Y.X. et al. Cancer Res. 68, 1905-1915 (2008)

4. Li, Y. et al. Oncogene 28, 3442-3455 (2009)

5. Mani, S.A. et al. Cell 133, 704-715 (2005)

6. Sabbah, M. et al. Drug Resist. Updat. 11, 123-151 (2008)

7. Weigelt, B. et al. Nat. Rev. Cancer 5, 591-602 (2005)

8. Janssen, J.W. et al. Oncogene 6, 2113-2120 (1991)

9. O'Bryan, J.P. et al. Mol. Cell Biol. 11, 5016-5031 (1991)

10. Liu, L. et al. Cancer Res. 69, 6871-6878 (2009)

11. Haas, M.J. SciBX 1(11); doi:10.1038/scibx.2008.250

\section{COMPANIES AND INSTITUTIONS MENTIONED}

BerGenBio A/S, Bergen, Norway

Exelixis Inc. (NASDAQ:EXEL), South San Francisco, Calif. GlaxoSmithKline plc (LSE:GSK; NYSE:GSK), London, U.K. Pfizer Inc. (NYSE:PFE), New York, N.Y.

Rigel Pharmaceuticals Inc. (NASDAQ:RIGL), South San Francisco, Calif. SuperGen Inc. (NASDAQ:SUPG), Dublin, Calif.

University of Bergen, Bergen, Norway

University of Colorado Denver, Denver, Colo.

Wyeth (NYSE:WYE), Madison, N.J. 